\title{
Creases in soft tissues generated by growth
}

\section{Citation}

Jin, Lihua, Shengqiang Cai, and Zhigang Suo. 2011. "Creases in Soft Tissues Generated by Growth." EPL (Europhysics Letters) 95 (6): 64002. https://doi.org/10.1209/0295-5075/95/64002.

\section{Permanent link}

http://nrs.harvard.edu/urn-3:HUL.InstRepos:41461216

\section{Terms of Use}

This article was downloaded from Harvard University's DASH repository, and is made available under the terms and conditions applicable to Other Posted Material, as set forth at http:// nrs.harvard.edu/urn-3:HUL.InstRepos:dash.current.terms-of-use\#LAA

\section{Share Your Story}

The Harvard community has made this article openly available.

Please share how this access benefits you. Submit a story.

Accessibility 


\title{
Creases in soft tissues generated by growth
}

\author{
Lihua Jin, Shengqiang Cai and Zhigang Suo ${ }^{(a)}$ \\ School of Engineering and Applied Sciences, Kavli Institute for Nanobio Science and Technology, \\ Harvard University - Cambridge, MA 02138, USA
}

received 19 June 2011; accepted 2 August 2011

published online 1 September 2011

PACS $46.32 .+\mathrm{x}-$ Static buckling and instability

PACS 87.16.A- - Theory, modeling, and simulations

PACS 87.17.Ee - Growth and division

\begin{abstract}
Soft tissues growing under constraint often form creases. We adopt the model of growth that factors the deformation gradient into a growth tensor and an elastic deformation tensor, and show that the critical conditions for the onset of creases take a remarkably simple form. The critical conditions are illustrated with tubes of tissues growing either inside a rigid shell or outside a rigid core. By comparing the critical conditions for the onset of wrinkles, we show that the creases are the preferred type of instability. Furthermore, deep creases in a tube are simulated by using the finite-element method, and the number of creases in the tube is estimated by minimizing the free energy.
\end{abstract}

Copyright (c) EPLA, 2011

Soft tissues often grow under constraint. For example, many tubular organs - arteries, airways, esophagi and intestines - grow under the constraint of a stiffer tissue, the smooth muscle [1,2]. Some tumors grow under the constraint of necrotic cores [3]. Even in the absence of any stiff tissues, soft tissues develop internal constraint when the growth is inhomogeneous [4,5]. Constrained growth can cause the soft tissues to undulate and fold. These mechanical instabilities have physiological and pathological consequences [3,6-11]. When the smooth muscle of airways shortens, the mucosa folds and obstructs the airways; the amount of obstruction increases in asthma due to the thickening of the airway walls $[6,7]$. Buckling can enable the invagination of embryos [9], and the primordial development of sunflowers [10]. Fingerprint patterns can result from the buckling of the layer of basal cells of the fetal epidermis [11]. The shape of a tumor can indicate its type [3].

Figure 1 illustrates several ubiquitous patterns of instability. For a soft material covered with a stiff thin layer, compression beyond a critical level leads to wrinkles [12]. Further compression will turn some of the wrinkles into folds $[13,14]$. For a soft material not covered with a stiff layer, however, compression beyond a critical level leads to creases, without forming wrinkles first [15-21]. While the wrinkles keep the surface of the material locally smooth, the folds and creases cause the surface to self-contact. The

(a) E-mail: suo@seas . harvard.edu

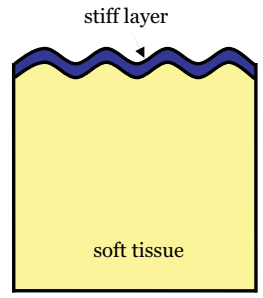

(a) wrinkles

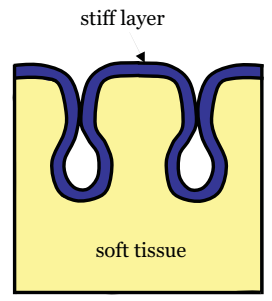

(b) folds

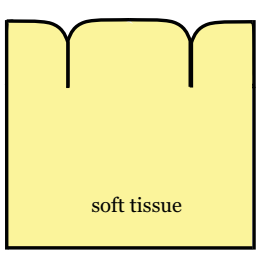

(c) creases
Fig. 1: (Color online) Schematic illustrations of three ubiquitous patterns of mechanical instability.

tip of a fold remains open due to the bending rigidity of the stiff layer, but the tip of a crease is sharp.

The onset of wrinkles corresponds to superposing a field of strain infinitesimal in amplitude, but finite in space. By contrast, the onset of creases corresponds to superposing a field of strain finite in amplitude, but infinitesimal in space. Consequently, the critical condition for the onset of wrinkles can be determined by a classical linear perturbation analysis, while the critical condition for the onset of creases cannot. Several recent papers have studied creases in soft materials caused by external forces [15-20]. This paper studies creases in tissues undergoing constrained growth. We show that the critical conditions for the onset of the growth-induced creases take a remarkably simple form. The critical conditions are illustrated with tubes of tissues growing either inside a rigid shell or outside a rigid 
core. Furthermore, deep creases in a tube are simulated by using the finite-element method, and the number of creases is estimated by minimizing the free energy.

As illustrated in fig. 2, we adopt a well-known model of growth [22]. A tissue is taken to be stress-free before the growth. An unconstrained and homogeneous growth changes the size of the tissue, but does not induce any stress. The stress-free growth is characterized by a growth tensor, $\mathbf{G}$, which maps any vector in the tissue in the stress-free state to a vector in the tissue after the growth by a stretch and a rotation. To model the constrained and inhomogeneous growth, we think of the tissue as many small elements, and name each element by its coordinate $\mathbf{X}$ when the tissue is in the stress-free state. The growth of the element is characterized by a growth tensor $\mathbf{G}(\mathbf{X})$, which may vary from one element to another. If only the stress-free growth tensor were considered, the neighboring elements would not fit together after the growth, but would have gaps or overlaps [23]. This incompatible, stress-free state after the growth is illustrated in fig. 2 . To make the elements compatible with one another, and to satisfy the constraint imposed by surrounding stiff tissues, each element deforms, which is characterized by a deformation tensor, $\mathbf{A}(\mathbf{X})$. The multiplication of the growth tensor and the deformation tensor gives the deformation gradient [22]:

$$
\mathbf{F}=\mathbf{A G}
$$

As illustrated in fig. 2, $\mathbf{G}(\mathbf{X})$ maps the tissue from the stress-free state before the growth to the stress-free state after the growth, $\mathbf{A}(\mathbf{X})$ maps the tissue from the stress-free state after the growth to the stressed state after the growth, and the deformation gradient $\mathbf{F}(\mathbf{X})$ maps the tissue from the stress-free state before the growth to the stressed state after the growth. While both $\mathbf{G}(\mathbf{X})$ and $\mathbf{A}(\mathbf{X})$ may be incompatible, their multiplication $\mathbf{F}(\mathbf{X})$ is compatible $[23,24]$.

The deformation gradient relates to the field of displacement in the usual way. Let an element of the tissue occupy a place of coordinate $\mathbf{X}$ when the tissue is in the stress-free state before the growth, and move to a place of coordinate $\mathbf{x}$ when the tissue is in the stressed state after the growth. The field of displacement in the tissue is represented by the function $\mathbf{x}(\mathbf{X})$. The constraint of the surrounding stiff tissues can be imposed by prescribing the values of the function $\mathbf{x}(\mathbf{X})$ for the elements of the tissue on the boundary. The deformation gradient is defined as

$$
F_{i K}=\partial x_{i}(\mathbf{X}) / \partial X_{K}
$$

We adopt the simplest version of the model, in which the field of the growth tensor $\mathbf{G}(\mathbf{X})$ is prescribed and is independent of the stress in the tissue. Furthermore, the deformation is assumed to be elastic and incompressible, $\operatorname{det}(\mathbf{A})=1$. The elasticity of the tissue is assumed to be

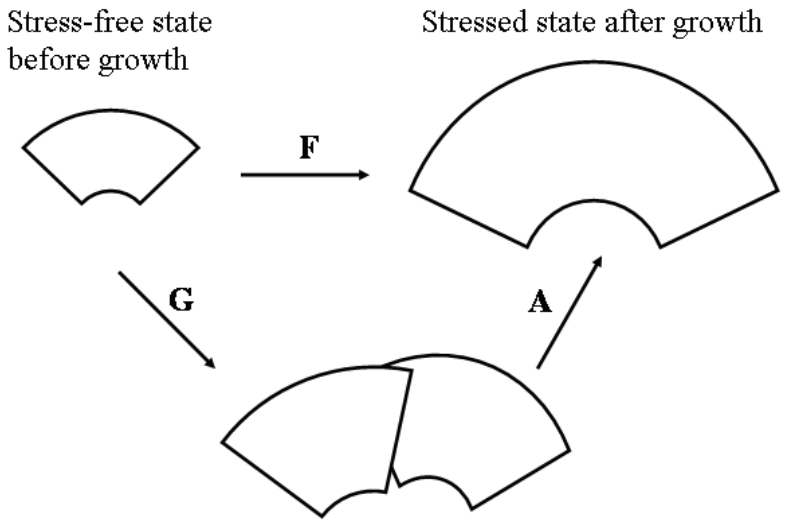

Stress-free state after growth

Fig. 2: A model of the growth of a tissue identifies three states: the stress-free state before the growth, the stress-free state after the growth, and the stressed state after the growth. Mapping from one state to another is accomplished by three tensors: the growth tensor $\mathbf{G}$, the deformation tensor $\mathbf{A}$, and the deformation gradient $\mathbf{F}$, which are related to each other by $\mathbf{F}=\mathbf{A G}$.

neo-Hookean and isotropic, so that the elastic energy of the tissue is

$$
\Pi=\int \frac{\mu}{2}\left[\operatorname{tr}\left(\mathbf{A}^{T} \mathbf{A}\right)-3\right] \operatorname{det}(\mathbf{G}) \mathrm{d} V,
$$

where $\mu$ is the shear modulus. The integral extends over the volume of the tissue in the stress-free state before the growth, and $\operatorname{det}(\mathbf{G})$ is the volume after the growth divided by that before the growth. If the body is subject to mechanical forces, the potential energy of the forces need be added to (3). The total free energy is minimized subject to the constraint $\operatorname{det}(\mathbf{A})=1$ and the boundary conditions of $\mathbf{x}(\mathbf{X})$.

Before creases set in, the surface of the tissue is smooth, and every element on the surface is in a well-defined state of strain. When the state of strain of an element reaches a critical condition, a crease sets in at this element - that is, the onset of each crease is autonomous [16,17]. For a neoHookean material subject to external forces, the critical conditions for the onset of creases have been calculated for general states of strain [16]. We now apply these conditions to tissues growing under constraint. Within the model of growth illustrated in fig. 2, the stress is developed in going from the stress-free state after the growth to the stressed state after the growth. Consequently, the critical conditions for the onset of creases obtained in [16] should be applied to the elastic deformation tensor $\mathbf{A}(\mathbf{X})$.

For an element $\mathbf{X}$ on the surface of the tissue, the three eigenvalues of $\mathbf{A}^{T} \mathbf{A}$ are denoted by $\alpha_{1}^{2}, \alpha_{2}^{2}, \alpha_{3}^{2}$ - that is, $\alpha_{1}, \alpha_{2}, \alpha_{3}$ are the principal elastic stretches. The surface of the tissue is assumed to be traction free, so that the direction normal to the surface is a principal direction of the stress tensor and, for a material of isotropic elasticity, 


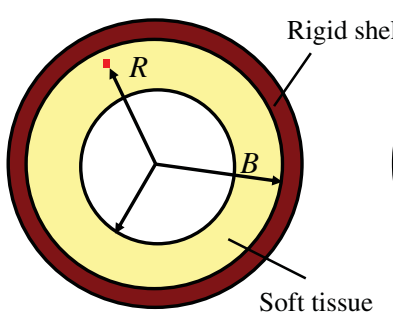

(a)

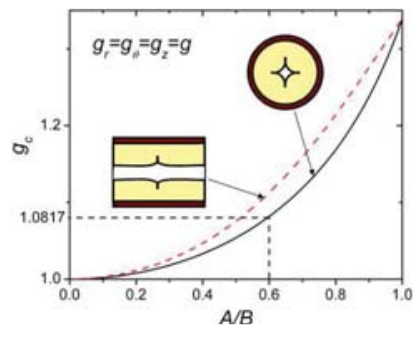

(c)

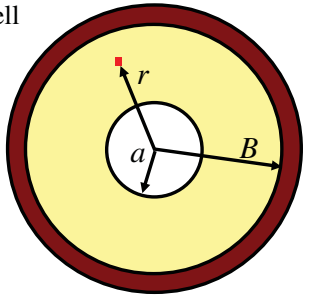

(b)

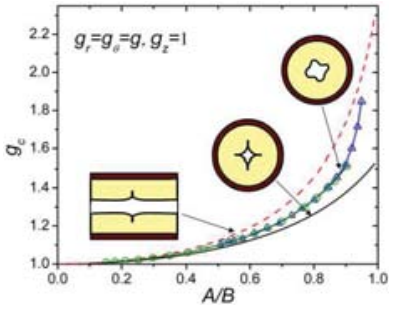

(d)
Fig. 3: (Color online) A tube of a tissue grows inside a rigid shell. (a) The stress-free state before the growth. (b) The axisymmetric stressed state after the growth. The critical growth ratio for the onset of creases as a function of $A / B$ for (c) isotropic growth and (d) an example of anisotropic growth. The solid lines are for circumferential creases, and the dashed lines are for longitudinal creases. In (d) the line with circles and triangles are the critical conditions for the onset of wrinkles. For the isotropic growth (c), when $A / B=0.6$, the critical growth ratio is $g_{c}=1.0817$.

is also a principal direction of the elastic deformation tensor. The other two principal directions are tangential to the surface of the tissue. We label the direction normal to the surface as direction 3 , and the other two principal directions as directions 1 and 2 . The critical condition for the onset of a crease normal to direction 1 is

$$
\alpha_{3} / \alpha_{1}=2.4,
$$

while the critical condition for the onset of a crease normal to direction 2 is

$$
\alpha_{3} / \alpha_{2}=2.4 \text {. }
$$

The value 2.4 was obtained for the incompressible neoHookean material by finite-element calculation in ref. [16], in which the critical condition for the onset of crease is defined as the condition when the elastic energy of creased state equals the smooth state. Creases will form first in the direction with the lower value in $\alpha_{1}$ and $\alpha_{2}$. If $\alpha_{1}=\alpha_{2}$, creases may form in any direction inside the surface.

We next illustrate the critical conditions with examples. Figure 3 shows a tube of a tissue growing in a rigid shell. In the stress-free state before the growth, the tube is of radii $A$ and $B$. In the stressed state after the growth, the tube is of radii $a$ and $B$. The rigid shell constrains the growth, and the soft tissue is perfectly bonded to the rigid shell, so that after the growth the outer radius and the length of the tube remain unchanged. Since the length of the tube is large compared to the radii, we assume that the tissue deforms under the plane-strain conditions. The growth tensor is assumed to be of the form $\mathbf{G}=\operatorname{diag}\left[g_{r}, g_{\theta}, g_{z}\right]$, where $g_{r}, g_{\theta}, g_{z}$ are the growth ratios in the radial, circumferential, and longitudinal directions of the tube. The growth is taken to be homogeneous, so that $g_{r}, g_{\theta}, g_{z}$ are constant independent of the position in the tissue.

Prior to the onset of creases, the growth causes the tube to undergo axisymmetric deformation. Consider an element of the tissue, at distance $R$ from the axis of the tube in the stress-free state before the growth. In the stressed state after the growth, the element moves to a place at distance $r$ from the axis of the tube. The function $r(R)$ specifies the field of displacement. Because the elastic deformation is taken to be incompressible, the increase in the volume of the tube is entirely due to the growth. Consider an annulus between the radii $B$ and $R$ in the stress-free state before the growth, and between the radii $B$ and $r$ in the stressed state after the growth. The growth increases the volume of the annulus by a factor $g_{r} g_{\theta} g_{z}$, so that

$$
B^{2}-r^{2}=g_{r} g_{\theta} g_{z}\left(B^{2}-R^{2}\right) .
$$

This expression specifies the field of displacement, the function $r(R)$. To prevent the tissue from interpenetrating after the growth, we require that $a>0$. Inserting this condition into (6), we obtain that $B^{2}>g_{r} g_{\theta} g_{z}\left(B^{2}-A^{2}\right)$. The deformation gradient takes the form $\mathbf{F}=\operatorname{diag}\left[\lambda_{r}, \lambda_{\theta}, \lambda_{z}\right]$, with the principal stretches being $\lambda_{r}=g_{r} g_{\theta} g_{z} / \lambda_{\theta}, \lambda_{\theta}=r / R$, and $\lambda_{z}=1$. The elastic deformation tensor takes the form $\mathbf{A}=\operatorname{diag}\left[\alpha_{r}, \alpha_{\theta}, \alpha_{z}\right]$, with the principal elastic stretches being $\alpha_{r}=\lambda_{r} / g_{r}$, $\alpha_{\theta}=\lambda_{\theta} / g_{\theta}, \alpha_{z}=\lambda_{z} / g_{z}$.

Following (4) and (5), the critical condition for the onset of circumferential creases is $\alpha_{r} / \alpha_{\theta}=2.4$ at the surface of the tube $R=A$, or

$$
g_{\theta}^{2} g_{z} /\left(\frac{B^{2}}{A^{2}}-g_{r} g_{\theta} g_{z}\left(\frac{B^{2}}{A^{2}}-1\right)\right)=2.4 .
$$

The critical condition for the onset of longitudinal creases is $\alpha_{r} / \alpha_{z}=2.4$ at the surface of the tube $R=A$, or

$$
g_{\theta} g_{z}^{2} / \sqrt{\frac{B^{2}}{A^{2}}-g_{r} g_{\theta} g_{z}\left(\frac{B^{2}}{A^{2}}-1\right)}=2.4 .
$$

In the limit $A / B \rightarrow 0$, any growth $g_{r} g_{\theta} g_{z}>1$ will cause very large compressive elastic strain at the inner surface of the tube, so that the critical condition for the onset of creases is $g_{r} g_{\theta} g_{z}=1$. In the limit $A / B \rightarrow 1$, the tube behaves like a flat layer constrained by a rigid substrate, so that the critical condition for the onset of the circumferential creases is $g_{\theta}^{2} g_{z}=2.4$, and the critical condition for the onset of the longitudinal creases is $g_{\theta} g_{z}^{2}=2.4$.

For isotropic growth, $g_{r}=g_{\theta}=g_{z}=g$, fig. 3(c) plots the critical growth ratio $g_{c}$ for the onset of creases as a function of $A / B$. The solid curve represents the critical condition for the onset of circumferential creases, and the dashed 


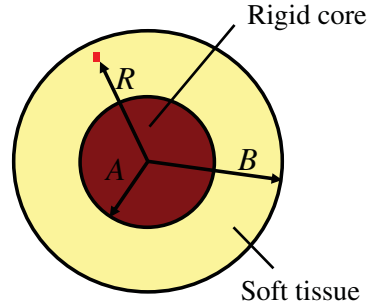

(a)

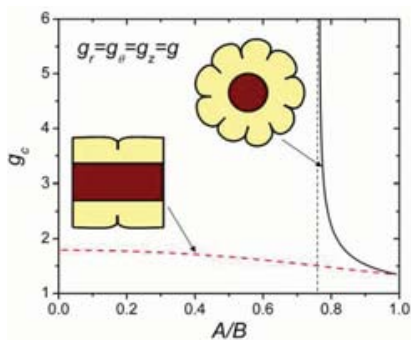

(c)

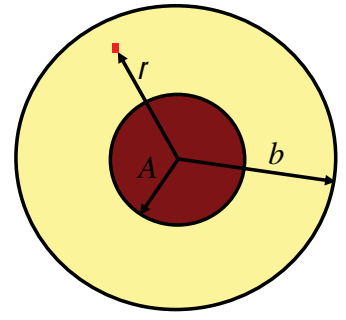

(b)

(d)

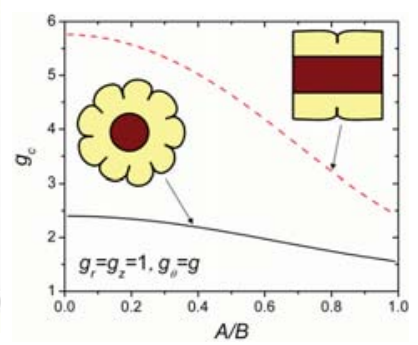

Fig. 4: (Color online) A tube of a tissue grows outside a rigid core. (a) The stress-free state before the growth. (b) The axisymmetric stressed state after the growth. The critical growth ratio for the onset of creases as a function of $A / B$ for (c) isotropic growth and (d) an example of anisotropic growth. The solid lines are for circumferential creases, and the dashed lines are for longitudinal creases.

curve represents the critical condition for the onset of longitudinal creases. For both types of creases, $g_{c}=1$ in the limit $A / B \rightarrow 0$, and $g_{c}=\sqrt[3]{2.4}$ in the limit $A / B \rightarrow 1$. Between the two limits, the growth ratio needed to initiate the circumferential creases is less than that needed to initiate the longitudinal creases.

As an example of anisotropic growth, consider the growth ratios $g_{r}=g_{\theta}=g$ and $g_{z}=1$. Figure $3(\mathrm{~d})$ plots the critical growth ratio $g_{c}$ for the onset of creases as a function of $A / B$. In the limit $A / B \rightarrow 1, g_{c}=\sqrt{2.4}$ for the onset of the circumferential creases and $g_{c}=2.4$ for the onset of the longitudinal creases. In this example, the growth needed to initiate the circumferential creases is less than that needed to initiate the longitudinal creases. Also included in fig. $3(\mathrm{~d})$ are the critical conditions for the onset of circumferential wrinkles obtained by the linear perturbation analysis (circles from [1] and triangles from [25]). A comparison of these results in fig. 3(d) indicates that the circumferential creases will set in, rather than the circumferential wrinkles.

Figure 4 illustrates a tube of a tissue growing outside a rigid core. The tube is of radii $A$ and $B$ in the stressfree state before the growth, and is of radii $A$ and $b$ in the stressed state after the growth. Prior to the onset of creases, the growth causes axisymmetric deformation in the tube. The field of deformation $r(R)$ is determined by

$$
r^{2}-A^{2}=g_{r} g_{\theta} g_{z}\left(R^{2}-A^{2}\right) .
$$

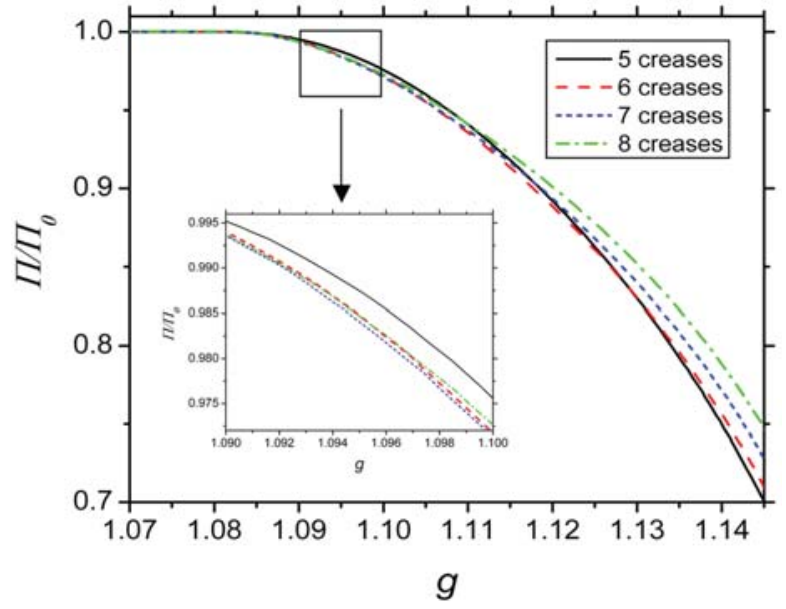

Fig. 5: (Color online) For a tube of a tissue growing inside a rigid shell, the ratio of the elastic energy of the creased state to that of the axisymmetric state, $\Pi / \Pi_{0}$, is plotted as a function of the growth ratio, $g$. The inset enlarges the squared region.

The critical condition for the onset of circumferential creases on the outer surface of the tube is

$$
g_{\theta}^{2} g_{z} /\left(\frac{A^{2}}{B^{2}}-g_{r} g_{\theta} g_{z}\left(\frac{A^{2}}{B^{2}}-1\right)\right)=2.4 .
$$

The critical condition for the onset of longitudinal creases on the outer surface of the tube is

$$
g_{\theta} g_{z}^{2} / \sqrt{\frac{A^{2}}{B^{2}}-g_{r} g_{\theta} g_{z}\left(\frac{A^{2}}{B^{2}}-1\right)}=2.4 .
$$

In the limit $A / B \rightarrow 0$, the rigid core becomes a thin needle, and the critical conditions for the onset of the circumferential and longitudinal creases become $g_{\theta} / g_{r}=$ 2.4 and $\sqrt{g_{\theta} g_{z}^{3} / g_{r}}=2.4$. In the limit $A / B \rightarrow 1$, the tube behaves like a flat layer constrained by a rigid substrate, which has been discussed above.

For the isotropic growth $g_{r}=g_{\theta}=g_{z}=g$, fig. 4(c) plots the critical level of growth $g_{c}$ for the onset of creases as a function of $A / B$. The critical condition for the onset of circumferential creases can only be satisfied when $A / B>\sqrt{7 / 12}$. Below this value, the rigid core does not provide sufficient constraint to cause circumferential creases on the outer surface of the tube. At all values of $A / B$, longitudinal creases will set in before circumferential creases. By contrast, in the case of anisotropic growth, $g_{r}=g_{z}=1, g_{\theta}=g$, circumferential creases can form in the entire range of $A / B$, and can form before longitudinal creases, fig. 4(d). In ref. [26], the swelling of a gel under constraint was studied in a similar geometry. When the core is much stiffer than the swelling gel, sharp creases were observed in the gel. The linear perturbation analysis in ref. [26] assumed infinitesimal strains from the smooth state, and corresponds to an analysis of wrinkling. By contrast, our analysis based on the result of 


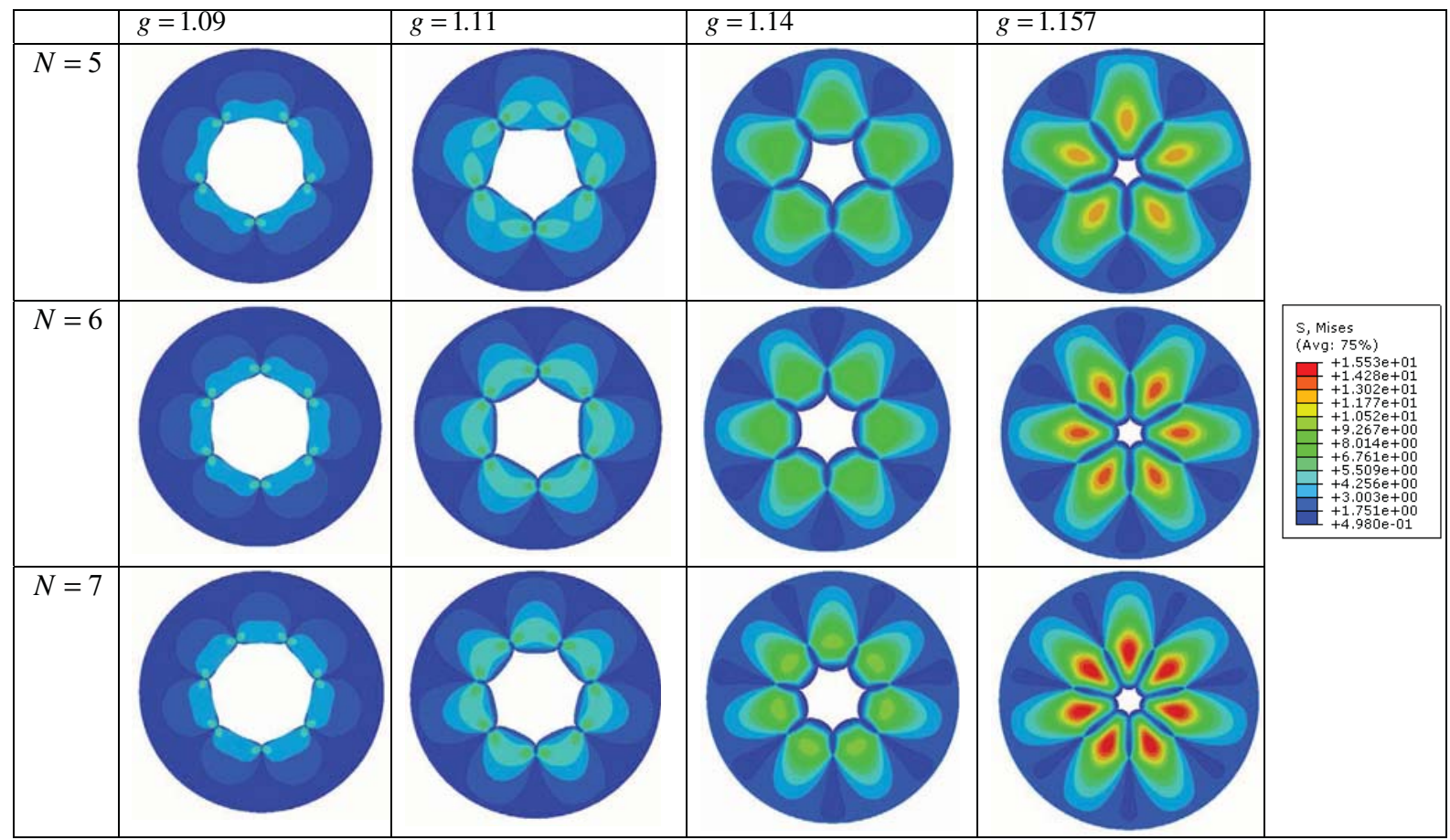

Fig. 6: (Color online) Deep creases develop from the surface of a tube of a tissue growing inside a rigid shell. Different numbers of creases are prescribed. The color indicates the level of the von Mises stress.

nonlinear finite-element method allows large strains from the smooth state, and corresponds to creases.

Because the state of strain is invariant everywhere on the surface of the tube under the axisymmetric deformation, every point on the surface reaches the critical condition to initiate a crease simultaneously. Consequently, the condition for the onset of creases does not determine the number of creases in a tube. In order to determine the number of creases, we go beyond the initiation of the creases and analyze deep creases. The analysis is carried out by using the commercial finite-element software ABAQUS, in which the growth of soft tissues can be simulated by thermal expansion. Plane-strain conditions are assumed. Symmetry is assumed such that if the number of creases is $N$, we only need to simulate one part of the tube within the angle $\pi / N$. Following ref. [19], we prescribe the nucleus of a crease by placing a defect, a quarter of a circle with small radius, on the surface of the tube. To eliminate the effect of the defect, its size is made much smaller than the thickness of the tube. At the same time, to resolve the field close to the defect, the size of the elements close to the defect is made much smaller than the size of the defect. The surface of the tube is allowed to self-contact.

As an example, consider a tube of a tissue growing inside a rigid shell, with $A / B=0.6$. The growth is taken to be isotropic, and the elasticity neo-Hookean. The finiteelement calculation determines the free energy per unit thickness of the tube as a function of the growth ratio,
$\Pi(g)$. The free energy per thickness of the axisymmetric deformation without creases can be calculated analytically, according to eq. (3):

$$
\begin{aligned}
\Pi_{0}= & \frac{\mu \pi g}{2}\left[B^{2}\left(1-g^{3}\right) \log \frac{g^{3}\left(A^{2}-B^{2}\right)+B^{2}}{A^{2}}\right. \\
& \left.+\left(2 g^{3}+1-3 g^{2}\right)\left(B^{2}-A^{2}\right)\right] .
\end{aligned}
$$

Figure 5 shows the ratio $\Pi / \Pi_{0}$ as a function of the growth ratio $g$ for several cases of prescribed numbers of defects. When $g$ is small, $\Pi / \Pi_{0}=1$, and the defects do not cause the surface to self-contact and become creased. Beyond a critical value $g_{c}=1.085$, however, $\Pi / \Pi_{0}<1$, and the defects cause the surface to self-contact and become creased. This critical value is consistent with the analytical prediction for the onset of creases $g_{c}=1.0817$ as shown in fig. 3(c). Right after the initiation, individual creases do not interact, so that more creases can release more energy. With further growth, the creases deepen and interact with one another. As shown in fig. 5, the energy in the tube with seven creases is the lowest when $g<1.1033$. After that the tube with six creases has the lowest energy until $g=1.1291$, and then the tube with five creases has the lowest energy. Figure 6 shows the creased states of the tube with different prescribed numbers of creases at different growth ratios. The color indicates the value of the von Mises stress. 
In summary, we obtain the critical conditions for the onset of creases caused by constrained growth. The critical conditions are illustrated with tubes of soft tissues growing under the constraint of either rigid outer shells or rigid inner cores. By comparing with the critical conditions for the onset of wrinkles, we show that creases are the preferred type of instability. Deep creases are simulated by using the finite-element method. The number of deep creases in a tube may be determined by minimizing the free energy.

$$
* * *
$$

This work is supported by the U.S. National Science Foundation through the grant CMMI-0800161, and by U.S. Army Research Office through the contract W911NF09-1-0476.

\section{REFERENCES}

[1] Li B., Cao Y. P. and Feng X. Q., J. Biomech., 44 (2011) 182.

[2] Li B., Cao Y. P., Feng X. Q. and Gao H., J. Mech. Phys. Solids, 59 (2011) 758.

[3] Dervaux J. and Ben Amar M., J. Mech. Phys. Solids, 59 (2011) 538.

[4] Goriely A. and Vandiver R., IMA J. Appl. Math., 75 (2010) 549.

[5] Ben Amar M. and Goriely A., J. Mech. Phys. Solids, 53 (2005) 2284.

[6] Wiggs B. R., Hrousis C. A., Drazen J. M. and Kamm R. D., J. Appl. Physiol., 83 (1997) 1814.

[7] Hrousis C. A. et al., J. Biomech. Eng., 124 (2002) 334.

[8] Yang W., Fung T. C., Chian K. S. and Chong C. K., J. Biomech., 40 (2007) 481.
[9] Pauchard L. and Couder Y., Europhys. Lett., 66 (2004) 667.

[10] Dumais J. and Steele C. R., J. Plant Growth Regul., 19 (2000) 7.

[11] Kucken M. and Newell A. C., Europhys. Lett., 68 (2004) 141.

[12] Bowden N. et al., Nature, 393 (1998) 146.

[13] Pocivavsek L. et al., Science, 320 (2008) 912.

[14] Sun J. et al., Folding wrinkles of a thin stiff layer on a soft substrate, to be published in Proc. R. Soc. A-Math. Phys. Eng. Sci.

[15] Hohlfeld E. and Mahadevan L., Phys. Rev. Lett., 106 (2011) 105702.

[16] Hong W., Zhao X. and Suo Z., Appl. Phys. Lett., 95 (2009) 111901.

[17] Cai S., Bertoldi K., Wang H. and Suo Z., Soft Matter, 6 (2010) 5770.

[18] Wong W. H., Guo T. F., Zhang Y. W. and Cheng L., Soft Matter, 6 (2010) 5743.

[19] Cai S., Chen D., Hayward R. C. and suo Z., Creasing instability of elastomer films, unpublished.

[20] CaO Y. and Hutchinson J. W., From wrinkles to creases in elastomers: The instability and imperfection-sensitivity of wrinkling, to be published in Proc. R. Soc. A-Math. Phys. Eng. Sci. (2011) DOI: 10.1098/rspa.2011.0384.

[21] Trujillo V., Kim J. and Hayward R. C., Soft Matter, 4 (2008) 564.

[22] Rodriguez E. K., Hoger A. and Mcculloch A. D., J. Biomech., 27 (1994) 455.

[23] Skalak R. et al., J. Math. Biol., 34 (1996) 889.

[24] Skalak R., in Proceedings of the IUTAM Symposium on Finite Elasticity, edited by CARLSON D. E. and SHIELD R. T. (Martinus Nijhoff, The Hague) 1982, pp. 347-355.

[25] Moulton D. E. and Goriely A., J. Mech. Phys. Solids, 59 (2011) 525.

[26] Dervaux J. et al., Phys. Rev. Lett., 107 (2011) 018103. 